\title{
Síndrome de Leriche: reporte de caso y revisión de literatura.
}

\author{
Johann Díaz Romero ${ }^{1}$, Alexis Idrobo Paredes ${ }^{2 *}$, Juan David Cruz Buriticá ${ }^{2}$, Sofía Guerrero Eraso ${ }^{3}$.
}

\begin{abstract}
${ }^{1}$ Médico Especialista en Medicina Interna, Universidad Libre Seccional Cali, Docente Pregrado en Medicina Interna, Facultad de Ciencias de la Salud, Universidad Libre Seccional Cali. Grupo de Investigación en Medicina Crítica y Trastornos Metabólicos. Miembro de la Asociación Colombiana de Medicina Interna. Cali-Colombia.

${ }^{2}$ Médico Interno, Universidad Libre Seccional Cali, Consorcio Clínica Nueva Rafael Uribe Uribe - Clínica DESA. Grupo de Investigación en Medicina Crítica y Trastornos Metabólicos. Cali-Colombia.

${ }^{3}$ Estudiante de Cuarto Año de Medicina, Universidad Libre Seccional Cali. Consorcio Clínica Nueva Rafael Uribe Uribe - Clínica DESA. Cali-Colombia.
\end{abstract}

\section{RESUMEN}

Introducción. Se describe el caso de un paciente que ingresa por una úlcera en miembro inferior derecho, con múltiples comorbilidades, con factores de riesgo para enfermedad ateroesclerótica que al examen físico presentaba claudicación intermitente, impotencia sexual y ausencia de pulsos en miembros inferiores, fue valorado integralmente por múltiples especialidades confirmando el diagnóstico de Síndrome de Leriche con reporte de angiotomografia, finalmente al paciente le fue amputado su miembro inferior derecho y se le dio egreso con recomendaciones y manejo multidisciplinario. Se resalta la importancia de un examen físico vascular periférico como método de sospecha diagnóstica y la oportuna intervención del paciente.

Palabras clave: Claudicación Intermitente, Enfermedad rterial periférica, Oclusión vascular, Síndrome de Leriche

\section{ABSTRACT}

Syndrome of Leriche: case report and review of literature.

Introduction. We present the case of a 78-year-old male, with multiple comorbidities, risk factors for atherosclerotic disease, who is admitted for ulcer in the right lower limb, to the physical examination presented intermittent claudication, erectile disffuntion and absence of pulses in the lower limbs. The patient was many different medical specialities evaluation and the diagnosis was confirmed with an angiotomography report, finally his right lower limb was amputated, discharged with recommendations and multidisciplinary management. The importance of a peripheral vascular physical examination as a method of diagnostic suspicion and the timely intervention of the patient is highlighted

Keywords: intermittent claudication, peripheral arterial disease, arterial occlusive diseases, Leriche syndrome.

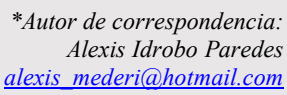

Como citar: Díaz Romero, J, Idrobo Paredes, A, Cruz Buriticá, JD, Guerrero Eraso, S. Síndrome de Leriche: reporte de caso y revisión de literatura. Revista Cuarzo 2019;25(2):28-31.

Recibido: 6 de julio de 2019 Aceptado: 24 de octubre de 2019 Publicado: 30 de diciembre de 2019 DOI: https://doi.org/10.26752/cuarzo.v25.n2.408 


\section{INTRODUCCIÓN}

$\mathrm{E}$ 1 síndrome de Leriche o síndrome de obliteración aortoiliaca se define como una oclusión de las ramas principales de la aorta abdominal por debajo de la salida de las arterias renales con compromiso de todo el árbol arterial incluyendo las arterias ilíacas y femorales (1). Se caracteriza por signos clínicos como impotencia sexual, ausencia de pulsos en arterias femorales, debilidad y entumecimiento en región lumbar, los glúteos, las caderas y extremidades inferiores (2).

El síndrome de Leriche fue descrito por Robert Graham en 1814 sin embargo, la definición actual fue descrita por el cirujano francés René Leriche en 1940 (2). Leriche vinculó la formación de trombos arteriales con una tríada de síntomas: claudicación, impotencia sexual y disminución de pulsos periféricos (1).

La incidencia y prevalencia no ha sido determinada dado que muchos de los pacientes que padecen esta patología son asintomáticos sin embargo, es una entidad relacionada con la edad avanzada, especialmente a partir de la sexta década de la vida y con un pico entre los 35 y 70 años siendo los principales afectados pacientes masculinos con antecedentes de enfermedad arterial periférica (EAP) o que cuentan con factores de riesgo como tabaquismo, hipertensión arterial, dislipidemia, obesidad y diabetes dado que en estos pacientes las placas ateromatosas localizadas en la aorta infrarrenal y las arterias ilíacas causan síntomas de obstrucción por bajo flujo sanguíneo $(1,2)$.

Se han reportado casos en mujeres con trastornos asociados con vasculitis como el Lupus Eritematoso Sistémico, la espondilitis anquilosante, entre otros (3).

El diagnóstico es principalmente clínico, siendo relevante la sospecha clínica para dirigir la exploración a los hallazgos más característicos de la enfermedad oclusiva aorto-iliaca (1).

La aortografía, la angioTAC o la arteriografía de los miembros inferiores son las principales pruebas para confirmar el diagnóstico, así como para valorar la extensión de la enfermedad, la gravedad de la oclusión, la presencia y extensión de circulación colateral y determinar las posibilidades de tratamiento (1).

El tratamiento es médico y quirúrgico, incluye cambios en los estilos de vida, prevención en factores de riesgo modificables, el manejo farmacológico y en cuanto al tratamiento quirúrgico se ha logrado realizar manejos a cielo abierto hasta el endovascular, aunque se describen pocos casos donde se realiza reperfusión mediante bypass y/o stent en el área comprometida de la aorta abdominal $(3,4)$.

\section{PRESENTACIÓN DEL CASO}

Paciente masculino de 78 años afrodescendiente proveniente de zona rural, quien refiere dolor y hormigueo en los miembros inferiores, consultó por cuadro clínico de 8 semanas de evolución aproximadamente, consistente en claudicación intermitente, parecía en miembros inferiores e impotencia sexual asociado a úlcera en dorso de glande y del muñón de pie derecho (Imagen 1.), con antecedentes de hipertensión arterial controlada, hiperlipidemia, diabetes mellitus noinsulinodependiente, falla renal crónica en reemplazo renal y mieloma múltiple sin control oncológico.

\section{Imagen 1. Úlcera en Dorso de Glande y del Muñón de Pie Derecho.}

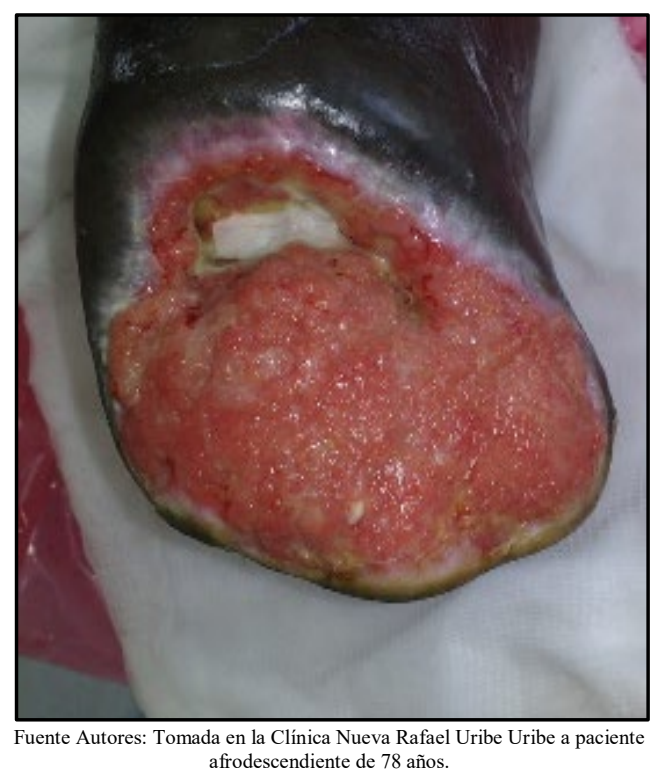

Al ingreso presenta signos vitales en rangos de normalidad, al examen físico con hallazgo de las úlceras ya mencionadas y especialmente en miembros inferiores frialdad, ausencia de pulsos y llenado capilar $>3$ segundos, se realiza medición de índice tobillo-brazo en 0.7 .

Es valorado inicialmente por Medicina Interna, Urología, Ortopedia y Cirugía Vascular quienes indican estudios de angiografía de abdomen y miembros inferiores donde se evidencia gran compromiso vascular arterial desde la aorta abdominal previo al nacimiento de las arterias iliacas comunes (Imagen 2) por lo cual Urología consideró manejo conservador $\mathrm{y}$ curaciones por terapia enterostomal de úlcera del glande con buena respuesta clínica, Cirugía Vascular y Ortopedia llevan el caso a junta médica quirúrgica donde se determina que la mejor opción terapéutica es la amputación infracondílea como método de control de síntomas y evitar progresión de isquemia periférica, Medicina Interna reajusta medicamentos para control de hipertensión arterial y diabetes mellitus, así como hiperlipidemia. 


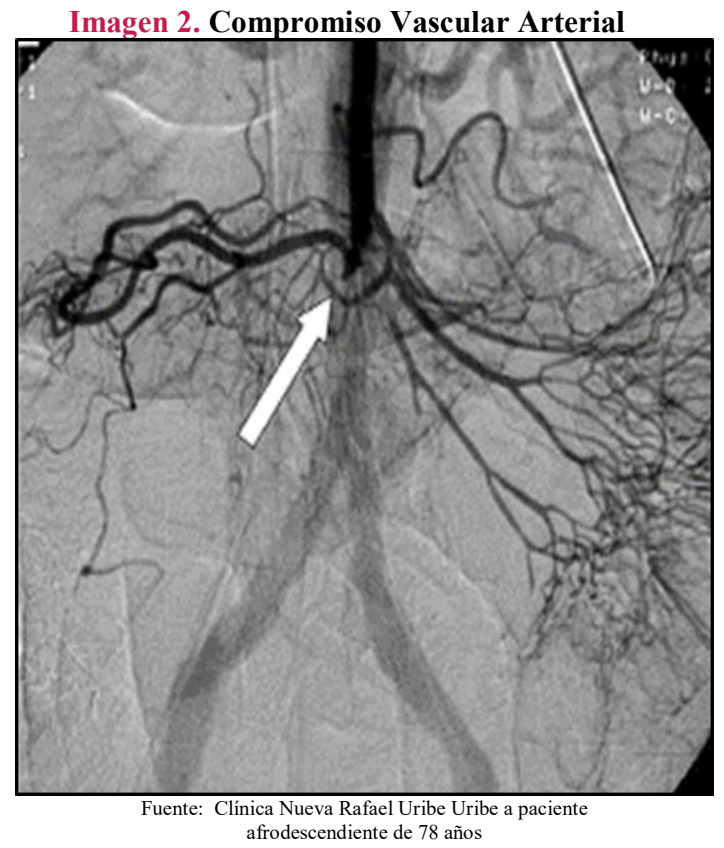

Finalmente, el paciente acepta la intervención quirúrgica la cual se realiza sin complicaciones (Figura 3), posteriormente cursa con buena evolución clínica, no presenta deterioro clínico de su condición por lo cual se da egreso con control ambulatorio por equipo multidisciplinario, lamentablemente paciente no asiste a controles y se pierde el seguimiento

Esquema 1. Comportamiento de las complicaciones por ocupación en adolescentes obstétricas, E.S.E Hospital la Divina Misericordia (2015).

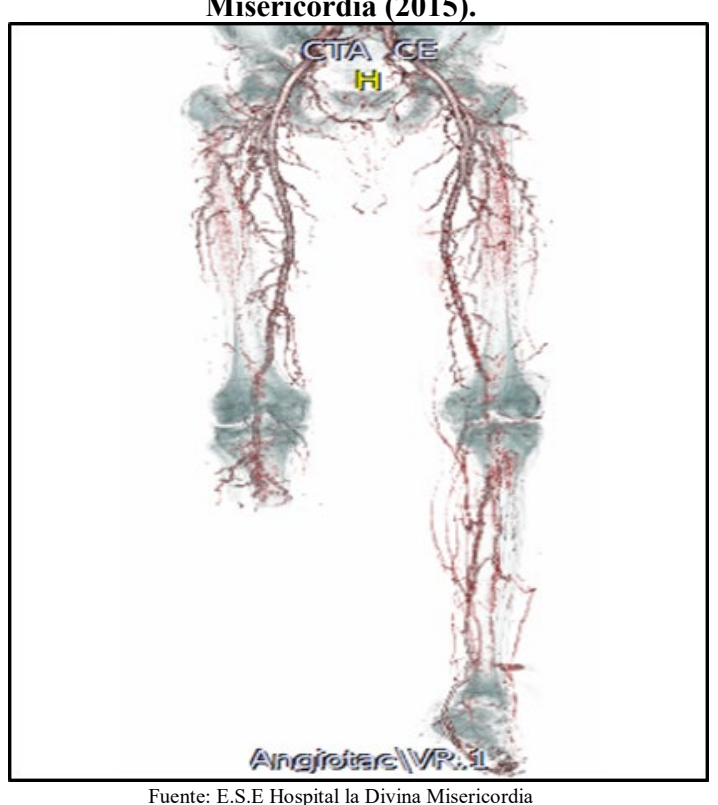

Fuente: E.S.E Hospital la Divina Misericordia

\section{DISCUSIÓN}

El síndrome de Leriche es una entidad subdiagnosticada teniendo en cuenta el gran número de pacientes asintomáticos debido al sedentarismo y al desarrollo de circulación colateral, la claudicación intermitente es su principal síntoma y su morbilidad es elevada dado el compromiso anatómico que genera en los miembros inferiores y su impacto incapacitante en la calidad de vida de los pacientes (2).

Fue descrito por primera vez en 1940 por el cirujano René Leriche, lo describió como una claudicación severa de los miembros inferiores, dolor en los glúteos, frialdad, palidez o cianosis en miembros inferiores e impotencia en varones, esta entidad se presenta principalmente en hombres, entre la tercera y sexta década de la vida (5). Existen factores de riesgo para esta entidad entre los cuales se encuentran hipertensión arterial, hiperlipidemia, diabetes mellitus y fumar, siendo la fisiopatología el resultado de una obstrucción ateromatosa de la aorta infrarrenal lo que se ve reflejado en atrofia muscular, mala cicatrización, frialdad e isquemia crítica de las extremidades ( 1 , $2,5)$.

En nuestro caso clínico se presenta a un paciente masculino de 78 años con antecedentes de hipertensión arterial, diabetes mellitus no-insulinodependiente, enfermedad renal crónica en etapa terminal en diálisis, síndrome metabólico y mieloma múltiple, con evidentes factores de riesgo para enfermedad arterial oclusiva crónica, presentando al examen físico la triada de claudicación intermitente, impotencia sexual y ausencia de pulsos, además de cursar con ulcera en pie derecho de difícil cicatrización y ulcera en el dorso de glande por lo cual fue inicialmente valorado en conjunto con Medicina Interna, Urología, Ortopedia y Cirugía Vascular para emitir concepto de amputación o terapia endovascular de salvamento de extremidades por lo que con el resultado de la angiotomografía se confirmó el diagnóstico y se definió conducta quirúrgica con amputación de la extremidad pues ya no contaba con circulación que permitiera cicatrización de su úlcera.

Existen numerosas herramientas para el diagnóstico, siendo de vital importancia la sospecha clínica, el uso del índice tobillo-brazo, ultrasonografía abdominal, angiografía y angiotomografía (5). En el caso de nuestro paciente se realizó sospecha diagnóstica por examen físico pues el paciente cursaba con isquemia de miembros inferiores y síntomas característicos de oclusión arterial, por lo cual fue estudiado con angiotomografía, pues esta representa una metodología diagnóstica no-invasiva, de gran utilidad que permite estudiar con gran precisión y detalle estructuras pequeñas lo que permite valorar el árbol arterial en el plano axial, coronal y sagital (6).

En cuanto al tratamiento se considera el endovascular como el tratamiento de primera línea por sus menores tasas de morbimortalidad sin embargo, se tiene como opción la cirugía a cielo abierto anatómica o extra-anatómica de acuerdo a las características clínicas del paciente, siendo desafortunado el desenlace con la amputación supracondílea del miembro inferior derecho en nuestro paciente, es por esta razón que se hace hincapié el valor del examen físico vascular periférico el cual con una sospecha clínica de obstrucción vascular se puede intervenir oportunamente pudiendo evitar destinos como la 
amputación de extremidades, dicho examen físico debe ser estratificado según la clasificación de Leriche-Fontaine (Tabla 1), es de especial importancia tener en cuenta este diagnóstico en aquellos varones hipertensos y diabéticos que manifiesten dolor en miembros inferiores e impotencia sexual (7).

Tabla 1: Clasificación Clínica de Leriche-Fontaine.

\begin{tabular}{|c|c|}
\hline Grado & Descripción \\
\hline Grado I & $\begin{array}{l}\text { Asintomático, detectable por índice tobillo-brazo } \\
<0.9\end{array}$ \\
\hline Grado IIa & $\begin{array}{l}\text { Claudicación intermitente no limitante para el modo } \\
\text { de vida del paciente }\end{array}$ \\
\hline Grado IIb & Claudicación intermitente limitante para el paciente \\
\hline Grado III & Dolor o parestesias en reposo \\
\hline Grado IV & Gangrena establecida, lesiones tróficas \\
\hline Grado III y/o IV & Isquemia crítica, amenaza de pérdida de extremidad \\
\hline \multicolumn{2}{|c|}{$\begin{array}{l}\text { Fuente: Tomado y adaptado de: Serrano Hernando F.J., Martín Conejero A. } \\
\text { Enfermedad arterial periférica: aspectos fisiopatológicos, clinicos y terapéuticos. } \\
\text { Rev Esp Cardial. } 2007 ; 60 \text { (9): } 969-82\end{array}$} \\
\hline & V. CONCLUSIONES \\
\hline
\end{tabular}

El síndrome de Leriche es una entidad frecuente en pacientes con riesgo cardiovascular y factores de riesgo para el desarrollo de enfermedad ateroesclerótica, principalmente en varones, subdiagnosticado dado que los pacientes son principalmente asintomáticos, se describe el caso de un paciente el cual tuvo como destino la amputación de su miembro inferior derecho dado que no fue intervenido oportunamente de su enfermedad, por lo cual se concluye y hace énfasis en el examen físico cardiovascular para evitar estos desenlaces y ofrecer una mejor calidad de vida a estos pacientes.

Conflicto de Interés: Los autores declaran NO tener conflicto de interés.

\section{REFERENCIAS}

1. Arnaiz García M. E., Arnaiz García J, Amado-Diago C. A., Diago Cuartero M.C., Arnaiz García A. M., García Martín A. Síndrome de obliteración aorto-iliaca: síndrome de Leriche. Med Gen y Fam. 2013; 2(4): 130-131

2. Martínez J, Díaz J. J., Luján V. P., Fernández M. R., Ramírez E. Enfermedad oclusiva aortoilíaca o síndrome de Leriche. Rev Colomb Cir. 2017; 32: 214-22

3. Muñoz Chávez J. C., Solarte Pineda H, Imbachi R. Caso clínico de interés: síndrome de Leriche. Rev Colomb Salud Libre. 2016; 11 (1): 57-61

4. Lee Wei-Jing, Cheng Yung-Ze, Lin Hung-Jung. Leriche syndrome. Int J Emerg Med. 2008; 1: 223

5. Assaad M, Tolia S, Zughaib M. Leriche sundrome: The inferior mesenteric artery saves the lower extremity. SAGE Open Medical Case Reports. 2017; 5: 1-3

6. Oviedo Gamboa I, Herbas Bernal R. I., Zegarra Sansiesteban W. Diagnóstico por tomografía del Síndrome de Leriche: reporte de un caso clínico. Gac Med Bol. 2013; 36 (2): 105107

7. Serrano Hernando F. J., Martín Conejero A. Enfermedad arterial periférica: aspectos fisiopatológicos, clínicos y terapéuticos. Rev Esp Cardiol. 2007; 60 (9): 969-. 